Este libro forma parte del acervo de la Biblioteca Jurídica Virtual del Instituto de Investigaciones Jurídicas de la UNAM

\title{
LA PANDEMIA COVID-19: ALGUNOS DILEMAS DEL CONFINAMIENTO PARA LAS MUJERES EN MÉXICO ${ }^{1}$
}

\author{
Amaranta MANRIQUE DE LARA RAMÍREZ \\ María de Jesús Medina ARellano
}

\begin{abstract}
SUMARIO: I. Introducción: violencia contra las mujeres y el \#QuédateEnCasa. II. Violencia doméstica y solidaridad. III. Trabajadoras de la salud: riesgos y seguridad. IV. Salud sexual y reproductiva: acceso al aborto libre y seguro. V. Apoyo en línea: acompañamiento y uso de apps. VI. Investigación y distribución de recursos escasos. VII. Reflexión final. VIII. Fuentes de consulta.
\end{abstract}

\section{INTRODUCGIÓN: VIOLENGIA CONTRA LAS MUJERES Y EL \#QUÉDATEENGASA}

El Día Internacional de las Mujeres de 2020 fue histórico en México. ${ }^{2}$ Decenas de miles de mujeres tomamos las calles y, al día siguiente, desaparecimos de ellas. Cada una a su manera, el 8 y 9 de marzo se visibilizó la violencia feminicida y la estructura que la sostiene, buscando generar un debate extendido, exigiendo pronunciamiento y compromiso de todos los actores clave. ${ }^{3}$ Pero mientras nosotras marchábamos en México, el mundo estaba ya comenzando a paralizarse por un virus que hasta ese momento había cobrado la vida de más de 60,000 personas, y en fechas recientes más

1 Este capítulo es una traducción modificada y ampliada de su versión original publicada en el Fournal of Bioethical Inquiry, bajo el título "The COVID-19 Pandemic and Ethics in Mexico Through a Gender Lens", con fecha 27 de agosto 2020. Una versión corta de este capítulo también fue preparada para la revista Género y Salud del Centro Nacional de Equidad de Género y Salud Reproductiva en México, en prensa.

2 Marcha del 8 y paro del 9 de marzo, El Universal, marzo de 2020, disponible en: https://wrere.eluniversal.com.mx/nacion/marcha-del-8-y-paro-del-9-de-marzo. Fecha de consulta: 22 de agosto de 2020.

3 "La revolución de las jacarandas", Nexos, abril de 2020, disponible en: https://wrere. nexos.com.mx/?p=47492. Fecha de consulta: 22 de agosto 2020. 
Este libro forma parte del acervo de la Biblioteca Jurídica Virtual del Instituto de Investigaciones Jurídicas de la UNAM

de 423, 349 vidas; ${ }^{4}$ lo anterior, obligo desde el inicio de la declaración de la pandemia a confinar personas y familias en sus hogares y abrumando los (de por sí frágiles) sistemas de salud. En esta situación inédita, las niñas, adolescentes y mujeres nos encontramos entre la espada y la pared de dos crisis de salud pública - la pandemia y la violencia de género - en un país donde el machismo es patrimonio nacional. ${ }^{5}$

Ante la rápida propagación de SARS-GoV-2, las medidas de distanciamiento resultan fundamentales. Paradójicamente, además de que el \#QuédateEnCasa se confronta con distintas realidades socioeconómicas, el aislamiento y confinamiento preocupa mucho en un país donde la violencia doméstica es la más incidente de las violencias hacia las mujeres, y gran parte de los feminicidios son perpetuados en contextos familiares, de pareja o amistad. ${ }^{6}$ En una pandemia, estas situaciones pueden exacerbarse. ${ }^{7}$ Por un lado, el estrés y la incertidumbre económica son disparadores; por el otro, el confinamiento aleja a las mujeres de sus redes de apoyo. Es imperante implementar programas de atención a la salud durante la pandemia con perspectiva de género para atender la problemática en el nuevo contexto: ${ }^{8}$ ¿Cómo puedo pedir ayuda si hay convivencia obligada con mi agresor? Por ejemplo, aplicaciones móviles podrían ser soluciones (aunque con limitaciones) si se habilitan para funcionar como botones de pánico. Es así, como a lo largo del texto señalamos las realidades desde miradas de mujeres en nuestro país, lo cual debe ser crucial para la adopción de políticas públicas en salud sexual y reproductiva durante la COVID-19, así también ha sido el llamado de más de 59 países en declaraciones públicas. ${ }^{9}$

\footnotetext{
4 Organización Mundial de la Salud (OMS). Who Coronavirus Disease (COVID-19) Dashboard, disponible en: https://covid19.who.int, fecha de consulta: 22 de agosto de 2020.

5 Htun, M, F. Jensenius, F. Aspirational laws as weak institutions: Legislation to combat violence against women in Mexico. En: The Politics of Institutional Weakness in Latin America, edited by D. M. Brinks, S. Levitsky, and M. V. Murillo, Cambridge, Cambridge University Press, en prensa.

6 UN Women, Global Database on Violence Against Women, disponible en: https://evaw-glo bal-database.unwomen.org/en/countries/americas/mexico. Fecha de consulta: 22 de agosto de 2020.

7 Capdevielle, P., Manrique de Lara Ramírez, A., Medina Arellano, M. de J. The COVID 19 pandemic and ethics through the eyes of women, Blog, BMf Journals-COVID-19, abril de 2020, disponible en: https://blogs.bmj.com/covid-19/2020/04/15/the-covid19-pandemic-andethics-through-the-eyes-of-women/. Fecha de consulta: 22 de agosto de 2020.

8 Capdevielle, P., Manrique de Lara Ramírez, A., Medina Arellano, M. de J., "Dilemas del \#QuédateEnCasa", Nexos, mayo de 2020, disponible en: https://wrere.nexos.com. $m x / ? p=47916$. Fecha de consulta: 13 de junio de 2020.

9 Klasing, A., "59 governments release joint statement on protecting SRHR and promoting Gender-responsiveness in the COVID-19 crisis", Human Rights Watch, 8 de mayo
} 
Este libro forma parte del acervo de la Biblioteca Jurídica Virtual del Instituto de Investigaciones Jurídicas de la UNAM

\section{VIOLENCIA DOMÉSTICA Y SOLIDARIDAD}

En el contexto mexicano y otras partes del mundo, la violencia estructural hacia la mujer es normalizada de muchas maneras y se manifiesta en los distintos niveles de la sociedad. El feminicidio ya cobraba miles de vidas de mujeres antes de la cuarentena, y esto no ha ido en disminución. ${ }^{10}$ Desafortunadamente, esta normalización es muchas veces representada por la prensa, y juega un rol crucial en este sentido, dado que las notas que cubren, por ejemplo, situaciones de feminicidio se hacen utilizando la tragedia de nuestros cuerpos para retratar la situación, sin embargo, el efecto colateral es la normalización del feminicidio y la perpetuación de las mujeres como grupo altamente vulnerable. ${ }^{11}$

El confinamiento, como señalamos, acentúa estas violencias. Otro ejemplo de esta situación desigualdad normalizada es en la repartición de labores no remuneradas de cuidado, basada en estereotipos machistas. Tan normalizada está, que hasta autoridades en nuestro país no dudaron en afirmar que las mujeres en casa podrían estar pendientes de la salud de las personas mayores, explicando que los varones suelen ser "más desprendidos". ${ }^{12}$ En la cuarentena, la mayoría de las mujeres serán responsables de: tareas domésticas incrementadas; cuidado de las infancias, incluyendo apoyo en su educación dado el cierre de escuelas; atención a familiares con alguna discapacidad física o mental; cuidado de personas ancianas; y, en su caso, atención a los mismos enfermos de GOVID-19. Las infancias tendrán efectos psicológicos adversos durante el confinamiento también, dado que las niñas y adolescentes también asumen tareas de cuidado en el apoyo a las madres. ${ }^{13}$ Algunas mujeres tendrán que lidiar también con la carga de trabajo profesional, considerando que de por sí viven

de 2020, disponible en: https://wrere.hrw.org/news/2020/05/08/governments-call-protection-sexual-and-reproductive-rights. Fecha de consulta: 13 de junio de 2020.

10 Lara Olmos, M., Ni una más: Feminicides in Mexico, 4 de abril de 2020, disponible en: https://justiceinmexico.org/femicidesinmexico/. Fecha de consulta: 12 de mayo de 2020.

11 Tiscareño-García, E., Miranda-Villanueva, O. M., "Victims and perpetrators of feminicide in the language of the Mexican written press", Comunicar. 2020; 28(63): 51-60. DOI: 10.3916/C63-2020-05.

12 Morales, A. et al., "Pide AMLO a mujeres y familias cuidar a los abuelitos ante coronavirus", El Universal, 24 de marzo de 2020, disponible en: https://wrere.eluniversal.com.mx/ nacion/politica/coronavirus-en-mexico-pide-amlo-mujeres-cuidar-abuelitos. Fecha de consulta: 29 de abril de 2020.

13 Wang, G. et al., "Mitigate the effects of home confinement on children during the COVID-19 outbreak", The Lancet, 395 (10228), 2020, pp. 945-947. 
Este libro forma parte del acervo de la Biblioteca Jurídica Virtual del Instituto de Investigaciones Jurídicas de la UNAM www.juridicas.unam.mx

mayor precariedad laboral y perciben salarios menores. ${ }^{14}$ Todo esto genera situaciones de agobio, cansancio crónico y desgaste mental, mismas que pueden afectar de manera permanente la salud emocional, mental y física de las mujeres, algunas veces llevándolas hasta el suicidio. ${ }^{15}$ Además, las corrientes bioéticas tradicionales tienden a enfocarse en valores individualistas, por ejemplo, la autonomía, ignorando otros principios bioéticos importantes, como son: solidaridad, compasión y valores comunitarios, que son esenciales en estos tiempos de pandemia. ${ }^{16}$

\section{TRABAJADORAS DE LA SALUD: RIESGOS Y SEGURIDAD}

Es importante resaltar que las mujeres conforman el 70\% de profesionales de atención a la salud en el mundo, de acuerdo con los datos de la Organización Mundial para la Salud (OMS); ${ }^{17}$ y además son mayoritarias en otros empleos con gran exposición al contagio (ej. empleadas de limpieza, cajeras en supermercados y farmacias). Es así, como creemos que en las conferencias de prensa las mujeres deberían estar más presentes, aquellas en puestos clave de enfrentamiento a la pandemia, puesto que son ejemplos o modelos por seguir para generaciones presentes y futuras, son nuestras heroínas. Sin embargo, tristemente no sorprende, pero es altamente ilustrativo, que la mayoría de las personas presentes en la conferencia de prensa sobre la pandemia COVID-19 en México son hombres "expertos", y esto se puede explicar porque la mayoría de los puestos directivos en los Institutos Nacionales de Salud y de la Secretaria de Salud son ocupados por hombres. ${ }^{18}$ De qué sirve

14 Scolese, A. et al., "Intimate Partner Violence Against Low-Income Women in Mexico City and Associations with Child School Attendance: A Latent Class Analysis Using Crosssectional Data", Maternal and Child Health Fournal, 2020; 24: 360-368.

15 Rojas-García, G. y M. P. Toledo González, "Paid domestic work: Gender and the informal economy in Mexico", Latin American Perspectives 45(1) 2017, pp. 146-162.

16 Medina-Arellano, M. de J., "Principios bioéticos y biojurídicos en tiempos de COVID-19: pacientes como vectores y víctimas", en Emergencia Sanitaria del COVID-19: Desde el derecho (I), N. González Martín (coord.), Mexico, UNAM, Instituto de Investigaciones Jurídicas, 2020, pp. 19-24, disponible en: https://wrere.juridicas.unam.mx/publicaciones/detalle-publicacion/151.

17 Organización Mundial de la Salud, OMS. Delivered by Women, Led by Men: A Gender and Equity Analysis of the Global Health and Social Workforce. Human Resources for Health Observer, 24, 2019, disponible en: https://wwre.who.int/hrh/resources/health-observer24/ en/. Fecha de consulta: 8 de abril de 2020.

18 Rivera-Romano, L. S. et al., "Gender imbalance in executive management positions at the Mexican National Institutes of Health”, Human Resources for Health, 18(1) 2020, pp. 21-32. DOI:10.1186/s12960-020-0463-4. 
Este libro forma parte del acervo de la Biblioteca Jurídica Virtual del Instituto de Investigaciones Jurídicas de la UNAM

que sean mayoría las heroínas en el sector salud, si siguen siendo subsumidas por la heteronormatividad patriarcal del ámbito médico. El colmo es que incluso el lenguaje que se utiliza es jerárquico, basado en estereotipos sexuales cuando se habla de las personas trabajadoras del sector salud, por ejemplo, constantemente se habla de médicos y enfermeras, lo cual contribuye a exponer la estructura de poder existente y dominante.

Finalmente, la falta de elementos de seguridad para las profesionales de salud, particularmente enfermeras, han sido atacadas, no solamente por el nocivo y descorazonado COVID-19, sino también por la sociedad desinformada, quien las ha atacado con una crueldad nunca vista, al menos en países como el nuestro, la India, Filipinas, incluso EUA, por mencionar algunos. ${ }^{19}$

\section{SALUD SEXUAL Y REPRODUGTIVA: ACGESO}

\section{AL ABORTO LIBRE Y SEGURO}

El destino prioritario de recursos y personal médico para atender la crisis puede afectar la salud sexual y reproductiva de las mujeres, en este país con altas tasas de violencia gineco-obstétrica. ${ }^{20}$ Aunque en nuestro país ya se emitió un lineamiento especial de atención para mujeres embarazadas, protegiéndolas también de contagio de COVID-19, ${ }^{21}$ nada se ha dicho sobre el acceso a servicios de emergencia a mujeres víctimas de violencia sexual, como medios seguros para interrumpir un embarazo producto de una violación. La misma preocupación existe sobre la disponibilidad de servicios no aplazables para terminar una gestación cuando existe una causal o, en el caso de la Ciudad de México y Oaxaca, incluso no solamente en situaciones de violencia sexual, sino cuando ésta no es deseada como parte de un derecho reproductivo fundamental. El derecho a la salud está vinculado al derecho a la vida, desarrollo de la personalidad, dignidad humana y libertad sexual y reproductiva, constituyendo derechos humanos que no pueden ser

19 McKay, D. et al., "Attacks against health-care personnel must stop, especially as the world fights COVID-19", The Lancet 395(10239) 2020: P1743-1745.

20 Calvo Aguilar, O. et al., "Obstetric violence criminalised in Mexico: a comparative analysis of hospital complaints filed with the Medical Arbitration Commission", BMF Sexual \& Reproductive Health, 46, 2020, pp. 38-45.

21 Secretaria de Salud. Lineamiento para la prevención y mitigación de COVID-19 en la atención del embarazo, parto, puerperio y de la persona recién nacida. México: Versión 1: 10 de abril de 2020, disponible en: https://wrerr.gob.mx/cms/uploads/attachment/file/546239/ Lineamiento_prevencion_y_mitigacion_de_COVID-19_en_el_embarazo_CNEGSR_1_.pdf. Fecha de consulta: 13 de junio de 2020. 
Este libro forma parte del acervo de la Biblioteca Jurídica Virtual del Instituto de Investigaciones Jurídicas de la UNAM www.juridicas.unam.mx

desbaratados por ninguna medida de excepcionalidad. Es importante señalar que la NOM-046 - una regulación técnica de observancia obligatoria implantada por el gobierno-, establece las medidas que se deben de adoptar para el acceso rápido y seguro a cualquier mujer víctima de violencia sexual, incluso si se trata de una niña menor de edad legal. De esta manera, estemos o no de acuerdo con la interrupción del embarazo, ante una situación de violencia en ningún Estado de la república debe de existir demora en el acceso a este derecho sexual y reproductivo, independientemente de las objeciones morales que presente el profesional de la salud.

Tristemente, en nuestro país durante estos tiempos de pandemia, en donde los feminicidios siguen en aumento y el abuso sexual también, en legislaturas locales como la de Guanajuato, se niegan a garantizar estos derechos, protegiendo la vida desde la concepción poniendo en peligro el acceso a mujeres a abortos libres y seguros dada la posible confusión a la que se pueden enfrentar las y los profesionales de la salud..$^{22}$ Estas discusiones ocurren a la par de fuertes declaraciones y llamados internacionales a los países alrededor del mundo a no manipular la crisis del COVID-19 y mucho menos aprovecharla para avanzar acciones en la restricción de acceso libre y seguro al aborto para las mujeres, es éste el llamado del Grupo de Expertas de Naciones Unidas contra la Discriminación de las Mujeres y Niñas. ${ }^{23}$

\section{APOYO EN LÍNEA: ACOMPAÑAMIENTO Y USO DE APLICACIONES MÓVILES}

El internet juega un papel clave en el seguimiento no solamente del control de la pandemia COVID-19, sino también en combatir la persistente violencia de género en nuestro país. De manera importante, el confinamiento o distanciamiento social ha exacerbado la violencia que se sufre en hogares, más aún donde los hogares se comparten con el perpetrador y en espacios de 5 por 10 metros cuadrados. Indudablemente, las aplicaciones móviles y el acceso al internet podrían ser herramientas adecuadas para garantizar apoyo psicológico, y así evitar no solamente violencia física, sino también

22 Agren D., "In two Mexican states, lawmakers vote down bills to legalize abortion", Crux, 28 de mayo de 2020, disponible en: https://cruxnow.com/church-in-the-americas/2020/05/ in-two-mexican-states-lawmakers-vote-down-bills-to-legalize-abortion/. Fecha de consulta: 30 de mayo de 2020.

23 United Nations Human Rights, Office of the High Commissioner, United States: Authorities manipulating COVID-19 crisis to restrict access to abortion, 27 de mayo 2020, disponible en: https://wrewe.ohchrorg/SP/Newesvents/Pages/DisplayNewes.aspx?NewsID=25907 ELang $I D=E$. Fecha de consulta: 30 de mayo de 2020 . 
Este libro forma parte del acervo de la Biblioteca Jurídica Virtual del Instituto de Investigaciones Jurídicas de la UNAM

suicidios. ${ }^{24}$ Medidas como estas han sido implementadas en México y otros países de América Latina. Un ejemplo en la Ciudad de México es el programa creado por la Comisión de Derechos Humanos de la Ciudad de México, que habilitó la línea \#CDHCMAmiga para atender y acompañar a víctimas de violencia de género durante la emergencia sanitaria por medio de texto o WhatsApp además de llamadas directas. ${ }^{25}$ Otro ejemplo es Argentina, el desarrollo de una aplicación gratuita por parte del Ministerio de las Mujeres, Géneros y Diversidad ${ }^{26}$ ha sido un recurso complementario de información, y aquellas mujeres que no tienen acceso a internet pueden llamar a otra amiga o familiar para que puedan hacer una llamada o mandar un mensaje mediante esta aplicación y directamente ayudar a la mujer que está en riesgo. Sin embargo, es importante recordar que, en temas de violencia de género, es importante la interseccionalidad. Es decir, reconocer que los factores socioeconómicos son un obstáculo incluso para acceder a un teléfono móvil o cualquier tecnología. Por lo tanto, los esfuerzos deben estar dirigidos aún en proporcionar acceso al internet y móviles a mujeres en situación de pobreza e indígenas, por ejemplo.

\section{INVESTIGACIÓN Y DISTRIBUCIÓN DE RECURSOS ESCASOS}

Más allá del riesgo de contraer COVID-19, el derecho a la salud de las mujeres está comprometido durante la pandemia. Por ejemplo, ante la contingencia sanitaria, algunos medios de comunicación en México han hecho declaraciones irresponsables e inexactas sobre la utilidad de algunos fármacos como la hidroxicloroquina, sin considerar que muchos de estos fármacos son esenciales para preservar la salud y vida de miles de mujeres, quienes son más propensas a desarrollar enfermedades crónicas como artritis reumatoide o lupus y que forman ya un grupo de riesgo ante COVID-19. ${ }^{27}$

24 Beleche, T., "Domestic violence laws and suicide in Mexico", Review of Economics of the Household 17(1), 2019, pp. 229-248.

25 Comisión de Derechos Humanos de la Ciudad de México. La CDHCM implementa línea de atención telefónica para mujeres víctimas de violencia. Boletín 60/2020, 15 de mayo de 2020, disponible en: https://cdhcm.org.mx/2020/05/la-cdhcm-implementa-linea-de-atencion-telefonica-para-mujeres-victimas-de-violencia/. Fecha de consulta: 14 de junio de 2020.

26 Ministerio de las Mujeres, Géneros y Diversidad. Medidas en Materia de Género y Diversidad en el Marco de la Emergencia Sanitaria, 15 de abril de 2020, disponible en: https://wwre.argentina.gob.ar/sites/default/files/medidasanteemergenciasanitaria_2.pdf. Fecha de consulta: 28 de abril de 2020.

27 Salgado-Galicia, N. A. et al., "The role of socioeconomic status in the susceptibility to develop systemic lupus erythematosus in Mexican patients", Clinical Rheumatology 39, 2020, pp. 2151-2161. 
Este libro forma parte del acervo de la Biblioteca Jurídica Virtual del Instituto de Investigaciones Jurídicas de la UNAM www.juridicas.unam.mx

La pandemia COVID-19, también nos ha enseñado que la mortalidad es más alta en varones, y también deberíamos explorar si se trata de condiciones generadas por el cumplimiento de masculinidades tóxicas. ${ }^{28} \mathrm{La}$ elaboración de cualquier política publica y de investigación en salud durante esta pandemia para enfrentar los retos que enunciamos debe de hacerse desde la perspectiva de género, dado que el entendimiento de las realidades intersecciones que enfrentamos las mujeres es esencial para entender impacto de la pandemia en nuestras vidas en México y el mundo. ${ }^{29}$ Para ejemplificar la incorporación de perspectiva de género en una política pública de salud presentamos el caso de la "Guía Bioética para Asignación de Recursos Limitados de Medicina Crítica en Situación de Emergencia”, en adelante Guía Bioética, publicada el 30 de abril de 2020 por el Comité de Ética de la Comisión Científica del Consejo de Salubridad en México. ${ }^{30}$ Es crucial reconocer que en algunas situaciones podemos vernos frente a una situación donde tuviéramos un ventilador y dos pacientes con la misma probabilidad de sobrevivir; en dichos casos la Guía Bioética mantiene que en un primer momento se deberá considerar la perspectiva de género conforme a la evidencia científica disponible, y en un segundo momento si uno de estos pacientes pertenece a grupos en situación de vulnerabilidad histórica. Si las consideraciones anteriores no desempatan la situación se recurriría al azar para decidir a quién otorgar el equipo médico escaso.

\section{REFLEXIÓN FINAL}

Finalmente, la pandemia ha logrado resaltar y exacerbar desigualdades y fallas a diferentes niveles. Aunque los paros nacionales de mujeres, $8 \mathrm{M}$ y $9 \mathrm{M}$, parecen de otra vida, las exigencias son vigentes ahora más que nunca. Estos temas sobre acceso a la salud, justicia y vida libre de violencia de las mujeres

\footnotetext{
28 The Conversation, COVID-19's deadliness for men is revealing why researchers should have been studying immune system sex differences years ago, 9 de junio de 2020, disponible en: https://theconversation.com/covid-19s-deadliness-for-men-is-revealing-why-researchersshould-have-been-studying-immune-system-sex-differences-years-ago-138767. Fecha de consulta: 13 de junio de 2020.

29 Papp, S. y Hersh, M. A., "Gender Lens for GOVID 19", Project Syndicate, 27 de marzo de 2020, disponible en: https://wrere.project-syndicate.org/commentary/covid19-response-requires-agender-lens-by-susan-papp-and-marcy-hersh-2020-03. Fecha de consulta: 28 de abril de 2020.

30 Consejo de Salubridad General, Guía Bioética para Asignación de Recursos Limitados de Medicina Crítica en Situación de Emergencia, 30 de abril 2020, disponible en: http:/ / wwre. csg.gob.mx/descargas/pdf/index/informacion_relevante/GuiaBioeticaTriaje_30_Abril_2020_7pm.pdf. Fecha de consulta: 22 de agosto de 2020.
} 
Este libro forma parte del acervo de la Biblioteca Jurídica Virtual del Instituto de Investigaciones Jurídicas de la UNAM

no deben quedar de lado, sino que deben asumirse como parte fundamental de cualquier plan de mitigación. Todas estamos preocupadas por la pandemia, pero no olvidemos que la violencia machista es el mal más fulminante contra nuestras vidas. Ningún gobierno deberá considerar estas situaciones como secundarias, por las mujeres en México, estamos seguras de que nunca olvidaremos, ni perdonaremos y desde todos los espacios: académico, activista, organizaciones de la sociedad, exigiremos justicia para todas.

\section{FUENTES DE CONSULTA}

Averbuch, M. 2020. "We'll Disappear": Thousands of Mexican Women Strike to Protest Femicide, The Guardian, 9 de marzo, https://wrere.theguar dian.com/world/2020/mar/09/thousands-mexican-womenprotest-violence-murdersfemicide-government-inaction.

Beleche, T., 2019, "Domestic violence laws and suicide in Mexico", Review of Economics of the Household 17(1): 229-248. DOI:10.1007/s11150-0179362-4.

Calvo Aguilar, O. et al., 2020, "Obstetric violence criminalised in Mexico: a comparative analysis of hospital complaints filed with the Medical Arbitration Commission”. BMF Sexual \& Reproductive Health 46: 38-45. DOI: 10.1136/bmjsrh-2018-200224.

El Universal, 2020, Women's Day 2020: Thousands of Women took over Mexico to Demand Justice and Equality through Massive Protests, 9 de marzo, disponible en: https://wrere.eluniversal.com.mx/english/womens-day2020-thousands-womentook-over-mexico-demand-justice-and-equality-throughmassive\#imagen-1.

Htun, M. y Jensenius, F., Forthcoming. Aspirational laws as weak institutions: Legislation to combat violence against women in Mexico, en The Politics of Institutional Weakness in Latin America, edited by D. M. Brinks, S. Levitsky, and M.V. Murillo. Cambridge: Cambridge University Press.

Klasing, A., 2020, Governments Call for Protection of Sexual and Reproductive Rights. Human Rights Watch, 8 de mayo, disponible en: https:// wwre.hrw.org/news/2020/05/08/governmentscall-protection-sexual-and-reproductive-rights.

MCKAY, D. et al., 2020, Attacks against health-care personnel must stop, especially as the world fights COVID-19, The Lancet, 395(10239): P1743-1745. 
Este libro forma parte del acervo de la Biblioteca Jurídica Virtual del Instituto de Investigaciones Jurídicas de la UNAM

Medina-Arellano, M. de J., 2020, Principios bioéticos y biojurídicos en tiempos de COVID-19: pacientes como vectores y víctimas in emergencia sanitaria del COVID-19: desde el derecho (I), N. González Martín, (coord.), México, UNAM, Instituto de Investigaciones Jurídicas, disponible en: https://wrere.juridicas.unam.mx/publicaciones/detalle-publicacion/151.

Ministerio de las Mujeres, Géneros y Diversidad, 2020, Medidas en Materia de Género y Diversidad en el Marco de la Emergencia Sanitaria. Last modified April 15, disponible en: https://wrww.argentina.gob.ar/sites/default/files/ medidasanteemergenciasanitaria_2.pdf.

Miranda-Ackerman, R. C. et al., 2019, Burnout syndrome prevalence during internship in public and private hospitals: a survey study in Mexico. Medical Education Online 24(1): 1593785. DOI:10.1080/10872981.2019.15 93785.

Morales, A. et al., 2020, "Pide AMLO a mujeres y familias cuidar a los abuelitos ante coronavirus", El Universal, 24 de marzo, disponible en: https://wrwreluniversal.com.mx/nacion/politica/coronavirus-en-mexico-pide-amlomujeres-cuidar-abuelitos.

NOM-046-SSA2-2005. 2009. Norma Oficial Mexicana NOM-046-SSA22005. Violencia familiar, sexual y contra las mujeres. Criterios para la prevención y atención, Diario Oficial de la Federación, 16 de abril, disponible en: http://wrew.cndh.org.mx/DocTR/2016/fUR/A70/01/fUR-20170331NOR19.pdf.

Olmos, M., 2020, Ni una más: Feminicides in Mexico, 4 de abril, disponible en: https://justiceinmexico.org/femicidesinmexico/.

Organización Mundial de la Salud (OMS), 2019, Delivered by Women, Led by Men: A Gender and Equity Analysis of the Global Health and Social Workforce. Human Resources for Health Observer 24, disponible en: https://www.who.int/hrh/resources/health-observer24/en/.

Organización Mundial de la Salud (OMS), 2020, Coronavirus (COVID-19), disponible en: https://covid19.who.int. Fecha de última consulta: 28 de abril de 2020 .

PADRÓN INNAMORATO, M. et al., 2017, No todo el trabajo es empleo: avances y desafios en la conceptualización y medición del trabajo en México. Ciudad de México: El Colegio Mexiquense, A. C.

Palacios-González, C., 2020, Coronavirus: los escenarios para México. Animal Político, 21 de marzo, disponible en: https://wrereanimalpolitico.com/ blog-invitado/coronavirus-losescenarios-para-mexico. 
Este libro forma parte del acervo de la Biblioteca Jurídica Virtual del Instituto de Investigaciones Jurídicas de la UNAM

PAPP, S. y M. Hersh, 2020, A Gender Lens for COVID-19. Project Syndicate, 27 de marzo, disponible en: https://wrere.project-syndicate.org/commentary/ covid19-response-requires-agender-lens-by-susan-papp-and-marcy-hersh-2020-03.

Rivera-Romano, L. S. et al., 2020, Gender imbalance in executive management positions at the Mexican National Institutes of Health. Human Resources for Health 18(1): 21-32. DOI:10.1186/s12960-020-0463-4.

Rojas-García, G. y TOledo GONZÁLez, M. P., 2017, Paid domestic work: Gender and the informal economy in Mexico. Latin American Perspectives 45(1): 146-162. DOI: 10.1177/0094582X17734545.

Salgado-Galicia, N. A. et al., 2020, The role of socioeconomic status in the susceptibility to develop systemic lupus erythematosus in Mexican patients. Clinical Rheumatology. DOI:10.1007/s10067-020-04928-5.

Scolese, A. et al., 2020, Intimate Partner Violence Against Low-Income Women in Mexico City and Associations with Child School Attendance: A Latent Class Analysis Using Cross-sectional Data. Maternal and Child Health Fournal 24: 360-368. DOI:10.1007/s10995-020-02877-8.

Secretaría de Salud, México, 2020, Guía Bioética para Asignación de Recursos Limitados de Medicina Crítica Situación de Emergencia, 11 de abril, disponible en: $h t t p: / /$ wrere.csg.gob. $m x /$ descargas/pdf/index/informacion_relevante/ GuiaBioeticaTriaje_30_Abril_2020_7pm.pdf.

Secretaría de Salud, México. 2020. Lineamiento para la prevención y mitigación de COVID-19 en la atención del embarazo, parto, puerperio y de la persona recién nacida, Mexico, 20 de abril, disponible en: https://wrere. gob.mx/cms/uploads/attachment/file/546239/Lineamiento_prevencion_y_mitiga cion_de_COVID-19_en_el_embarazo_CNEGSR_1_.pdf.

Tiscareño-García, E. y Miranda-Villanueva, O. M., 2020, Victims and perpetrators of feminicide in the language of the Mexican written press. Comunicar 28(63): 51-60. DOI:10.3916/C63-2020-05.

UrRUtia, A. y JiMÉneZ, N., 2020, Crecen feminicidios en lo que va del año, reporta Durazo. La fornada, 20 de julio, disponible en: https://wrere.jornada. com.mx/ultimas/politica/2020/07/20/crecen-feminicidios-enlo-que-va-del-anoreporta-durazo-2781.html.

WANG, G. et al., 2020, Mitigate the effects of home confinement on children during the COVID-19 outbreak. The Lancet 395(10228): 945-947.

Xantomila, J., 2020, ONU: Feminicidios en México crecieron diariamente de 7 a 10 en tres años. La fornada, 5 de marzo, 2020, disponible en: https:// wrere.jornada.com.mx/ultimas/sociedad/2020/03/05/onu-feminicidios-en-mexico-crecieron-de-7-a-10-diarios-en-tres-anos-8647.html. 